Revue d'histoire de l'Amérique française

REVUE D.HISTOIRE DE L'AMÉRIQUE FRANÇAISE

\title{
Une correspondance inédite de Joseph-Louis Painchaud (1819-1855)
}

\section{Louis Painchaud}

Volume 41, numéro 2, automne 1987

URI : https://id.erudit.org/iderudit/304552ar

DOI : https://doi.org/10.7202/304552ar

Aller au sommaire du numéro

Éditeur(s)

Institut d'histoire de l'Amérique française

ISSN

0035-2357 (imprimé)

1492-1383 (numérique)

Découvrir la revue

Citer cette note

Painchaud, L. (1987). Une correspondance inédite de Joseph-Louis Painchaud (1819-1855). Revue d'histoire de l'Amérique française, 41(2), 233-236.

https://doi.org/10.7202/304552ar d'utilisation que vous pouvez consulter en ligne.

https://apropos.erudit.org/fr/usagers/politique-dutilisation/ 


\section{NOTE DE RECHERCHE \\ UNE CORRESPONDANCE INÉDITE DE JOSEPH-LOUIS PAINCHAUD (1819-1855)}

LOUIS PAINCHAUD

Faculté de théologie Université Laval

Pour marquer le 50e anniversaire de la Société de Saint-Vincentde-Paul à Québec, Mgr Horace Têtu publiait en 1897 un volumineux ouvrage auquel étaient annexés des extraits de dix lettres de JosephLouis Painchaud, dont huit adressées à ses parents ${ }^{1}$. L'excerpteur avait alors choisi de publier certains passages qui mettaient en lumière le zèle et la charité de celui que l'on s'entend à considérer comme le fondateur de cette société charitable au Canada.

En 1919, C.-J. Magnan, alors président de la Société, utilisa ces mêmes lettres pour préparer une brochure édifiante qu'il consacra au docteur Painchaud afin de souligner le centenaire de sa naissance ${ }^{2}$. Le texte de cette brochure ne contient toutefois aucune allusion à cette correspondance à laquelle personne ne s'intéressa plus, mais qui fut conservée dans la famille Painchaud. Ces lettres présentent pourtant un intérêt certain pour l'histoire religieuse et sociale du Canada français car elles mettent en lumière l'univers religieux de ce que l'on appellerait aujourd'hui un laïc engagé ${ }^{3}$ et que Magnan, en 1919, proposait comme un modèle de «chrétien éclairé» et de «catholique d'action» en le comparant à Frédéric Ozanam ${ }^{4}$.

Joseph-Louis Painchaud est né à Québec le 12 juin $1819^{5}$. Il était le fils de Joseph Painchaud ${ }^{6}$, médecin, et de Geneviève Parant. Il fit

\footnotetext{
1 H. Têtu, Les noces d'or de la Société Saint-Vincent-de-Paul de Québec (Québec, Pruneau et Kirouac, 1897), 328-347. $16 \mathrm{p}$.

C.-J. Magnan, Le docteur Joseph Painchaud (Montréal, L'Oeuvre des Tracts, 1919),

On sait comment cet aspect de notre histoire religieuse est resté encore relativement peu étudié jusqu'à nos jours. Cette carence a déjà été soulignée par Pierre Savard, «Un type de laïc au Canada français traditionnel: le journaliste catholique», P. Hurtubise et al., Le laïc dans l'Église canadienne de 1830 à nous jours (Montréal, Fides, 1972), 93-102.

4 J.-C. Magnan, op. cit., 16.

5 Voir pour plus de détails, L. Painchaud, «Painchaud, Joseph-Louis», Dictionnaire biographique du Canada, 8: 751-752 et les indications bibliographiques.

6 Voir C.-M. Boissonnault, «Painchaud, Joseph», Dictionnaire biographique du Canada, 10: 618-619 et les nombreux articles que lui a consacrés le docteur Sylvio Leblond.
} 
ses études au Petit Séminaire de Québec qu'il quitta en 1840 . Il voulut alors se faire prêtre mais ne le put en raison d'une infirmité à une jambe. Il fit alors l'apprentissage de la médecine auprès de son père et du docteur James Douglas, puis il se rendit à Paris pour y parfaire sa formation. C'est alors qu'il connut la Société de Saint-Vincent-de-Paul, fondée en cette ville par Frédéric Ozanam en 1833, et qu'il devint membre de la Conférence de Saint-Séverin. De retour à Québec, il fut sollicité à l'automne 1846 par messire Charles-François Baillargeon, alors curé de la cathédrale, pour rendre cette société qui existait déjà à Québec, plus conforme à son modèle parisien. Painchaud ne se contenta pas de ce rôle de «réformateur». Il fit tant et si bien que le 7 mars 1847, Québec comptait neuf conférences, dont une à l'Hôpital de la Marine et des Émigrants où il était médecin résident.

En 1849, après qu'il eût quitté cet hôpital pour des raisons demeurées obscures ${ }^{7}$, et semble-t-il, pour mettre à exécution un voeu qu'il avait fait, Joseph Painchaud se donna corps et biens à Mgr Modeste Demers qui venait d'être sacré évêque de Vancouver. En septembre, il s'embarqua donc pour Paris où il devait introduire l'évêque missionnaire dans la bonne société afin d'amasser des fonds et de faire du recrutement pour la Mission. Il prévoyait y rester quelques semaines mais les circonstances politiques l'y retiendront jusqu'en juillet 1851, date à laquelle il partit pour Vancouver, destination qu'il n'atteindra jamais. Après avoir connu diverses mésaventures, Joseph Painchaud serait mort au Mexique en 1855 alors qu'il y exploitait une mine d'argent.

C'est pour respecter une promesse faite à sa mère avant de quitter Québec que Joseph Painchaud écrivit à chaque quinzaine une lettre à destination de la maison paternelle sise au coin de la rue des Pauvres (l'actuelle côte du Palais) et de la rue des Baraques (ruelle de l'Arsenal).

La plupart des quarante lettres qui ont été conservées (sur un total probable de 43$)^{8}$ sont divisées en sections qui sont adressées, généralement selon l'ordre suivant, à son père, puis à sa mère et à chacun de ses frères et soeurs, Geneviève (1816-1883) épouse du docteur Pierre Baillargeon, qui était alors domiciliée rue Sainte-Ursule, Joséphine (1818-1862) en religion mère Saint-André, religieuse à l'Hôtel-Dieu, Antoinette (1821-1862) résidant à la maison paternelle et toujours en quête d'un mari à 28 ans $^{9}$, Édouard (1825-1861) célibataire, qui semble

\footnotetext{
7 Il quitta l'Hôpital de la Marine en octobre 1848, quelques mois après avoir eu à comparaître devant une commission d'enquête (mai 1848) pour des motifs que nous ignorons.

8 Il s'agit de 89 feuillets de formats divers, la plupart de papier oignon, écrits recto, verso, pour un total de 178 pages.

9 Elle épousera le 26 juillet à Notre-Dame de Québec, Édouard-R. Fréchette.
} 
en proie à des troubles émotifs ${ }^{10}$ et enfin Antoine, le cadet de la famille dont l'avenir professionnel n'est pas encore fixé (1827-1903) ${ }^{11}$.

Son père était un «lectureur» à la mode, comme on disait à l'époque, ce qui désolait son fils qui n'appréciait guère cette activité. Sa mère était fort dévote et, semble-t-il, d'une grande charité qui s'exerçait surtout dans des visites et des secours dispensés à des familles pauvres qui ne manquaient pas à Québec. Des deux côtés, cette famille entretenait d'étroites relations aussi bien avec les milieux clérical, religieux et médical de Québec où elle avait des représentants nombreux et bien placés $^{12}$, qu'avec des familles qui comptèrent bon nombre de médecins, avocats, négociants et hommes politiques ${ }^{13}$.

Ce corpus de quarante lettres ouvre tout à la fois sur ce monde de la petite bourgeoisie des professions libérales de Québec et sur le Paris agité de la IIe République en même temps qu'il nous dévoile l'univers parfois étonnant de ce jeune médecin idéaliste et religieux à l'excès. Il se passionne pour la figure de Louis XVII, donne un écho enthousiaste à la ruée vers l'Eldorado californien et attend une fin du monde imminente tout en fustigeant Eugène Sue en particulier et ce qu'il appelle la «Ruine publique» en général. On y découvre pêle-mêle sa perception de l'actualité parisienne et les soucis qu'il se fait pour les différents membres de sa famille, qu'il s'agisse de l'avenir professionnel de son frère cadet ou du salut éternel de son père, tous deux trop frivoles à son goût. Il fait largement état de ses pratiques de dévotion et y exprime ses sentiments religieux, son engouement pour les apparitions de la Salette et de Notre-Dame-des-Victoires, sa perception du mariage, de la mort, de l'éducation des enfants et du missionnariat, le tout livré sans art ni apprêt.

Ce n'est toutefois pas uniquement par ce qu'elles nous révèlent de leur auteur que ces lettres sont intéressantes. Elles reflètent aussi indirectement la perception que son propre milieu avait de lui. Ainsi, il

10 Plusieurs lettres font allusion à ses «aberrations». Une lettre de lui, datée de Blandford, le 11 avril 1855 et adressée à l'abbé Hospice Verreau, est conservée aux archives du Séminaire de Québec. (Fonds Verreau 32 N0367). Il y paraît tourmenté, en proie à l'indécision et à l'angoisse.

11 Il épousera Élisabeth Le Bouthillier à Gaspé en 1855. C'est dans sa descendance que ces lettres ont été conservées.

12 Marie-Louise Painchaud, sa tante, était religieuse à l'Hôtel-Dieu. Elle y fut élue supérieure de 1840 à 1843 , puis de 1849 à 1855. Quant à son oncle Antoine Parant (1784-1855), il était prêtre au Séminaire de Québec où il exerça et cumula parfois les charges de supérieur et de procureur. Son oncle Joseph Parant (1796-1856) était médecin à l'Hôpital Général avec son beaufrère Joseph Painchaud. Il a également été pendant quelques années le médecin du Monastère des Ursulines et, de 1825 à 1847 , celui de l'Hôtel-Dieu. Il fut aussi commissaire à l'Hôpital de la Marine. Quant à Joseph Painchaud père, outre sa pratique privée et son office à l'Hôpital Général, il fut médecin à l'Hôpital de la Marine et des Émigrants de 1820 à 1871 et médecin attitré du Séminaire de Québec. L'un et l'autre exercèrent de nombreuses fonctions au sein de leur profession.

13 Les Pelletier, les Baillargeon, les Landry et les Têtu. 
semble bien que son père ait peu apprécié que son fils se soit donné corps et biens à la mission de Vancouver et une querelle épistolaire à propos des problèmes financiers de Joseph-Louis et du refus de son père d'y pourvoir est sans doute la cause de la disparition de certaines lettres de décembre-janvier 1850-1851. En outre, Joseph-Louis proteste, dans une de ses lettres, contre certaines critiques qu'aurait formulées à son égard l'abbé Pierre-Télesphore Sax après qu'il l'eût rencontré à Paris en 1850. Il réagit également à diverses remarques faites à son sujet par des membres de sa famille ou des connaissances.

Ainsi, ce corpus nous révèle, derrière l'image du «chrétien héroïque» peinte par C.-J. Magnan en 1919, un être fortement contrasté, intensément religieux mais aussi empreint de profondes contradictions, diversement reçu dans son milieu familial et social. Il s'agit donc ici d'un document de première importance en ce qu'il nous fait connaître et comprendre non seulement son auteur, mais aussi le milieu qui fut le sien et la façon dont il y fut perçu.

Les 89 feuillets qui constituent ce corpus sont généralement bien conservés, quelques-uns toutefois sont endommagés ou amputés de leur début, de sorte qu'il a fallu recourir à la critique interne et externe pour reconstituer l'ordre des lettres, dont les feuillets avaient été mêlés, et dater celles qui ne le sont plus.

Grâce à des subventions du fonds FCAR (volet ACSAIR), de la Fondation Gérard-Dion et du CEGEP de Sainte-Foy, il a été possible à une équipe ${ }^{14}$ de déchiffrer l'ensemble du manuscrit, d'en établir le texte et de les mettre sur support informatique. Des index des noms propres et du vocabulaire religieux ont également été constitués et saisis sur support informatique. Un travail d'analyse et d'explication de l'ensemble du corpus est en cours en vue d'en préparer une édition intégrale mais le texte et les index sont déjà accessibles sous certaines conditions.

\footnotetext{
14 Alain Bouchard, Roland Legendre et Louis Painchaud, professeurs de sciences religieuses et d'histoire au département des sciences humaines du CEGEP de Sainte-Foy.
} 